\title{
Electrophoresis of concentrated mercury drops
}

\author{
Eric Lee, Jin-Kan Hu, and Jyh-Ping Hsu * \\ Department of Chemical Engineering, National Taiwan University, Taipei, Taiwan 10617, ROC
}

Received 16 April 2002; accepted 22 October 2002

\begin{abstract}
The electrophoretic behavior of concentrated monodispersed, positively charged mercury drops is investigated theoretically. The present study extends previous analyses by considering arbitrary surface potentials, double-layer polarization, and the interaction between adjacent double layers. The coupled equations describing the spatial variations in the flow field, the electric field, and the concentration field are solved by a pseudo-spectral method. For a low surface potential $\phi_{r}$, the mobility increases monotonically with $\kappa a ; \kappa$ and $a$ are respectively the reciprocal Debye length and the radius of a mercury drop. For medium and high $\phi_{r}$, the mobility curve has a reflection point, which arises from the interaction of adjacent double layers, for $\kappa a$. Also, if $\phi_{r}$ is high, the mobility curve may exhibit a local minimum as $\kappa a$ varies. This phenomenon is pronounced if the concentration of the dispersed phase is high. If the double layer is thick, the mobility increases with $\phi_{r}$, and the reverse is true if it is thin. We show that the higher the concentration of the dispersed phase the smaller the mobility, and as $\kappa a$ becomes large the mobility approaches a constant value, which is independent of the concentration of the dispersed phase. The mobility of mercury drops is larger than that of the corresponding rigid particles.
\end{abstract}

(c) 2003 Elsevier Science (USA). All rights reserved.

Keywords: Mercury drops; Electrophoresis; Pseudo-spectral method; Double layer polarization; Mobility

\section{Introduction}

The electrokinetic phenomenon is one of the important branches of colloidal science. The phenomenon, which includes electrophoresis, electroosmosis, sedimentation potential, streaming potential, and electric conductivity, is closely related to the properties of a particle, in particular, the charged conditions on its surface. Unfortunately, this can only be characterized approximately by the electrical potential or charge density on the shear plane of the double layer surrounding a particle, and the exact surface properties remain unknown. Furthermore, limited by the available experimental apparatus, the reliability of the experimental data gathered often deserves further observation.

The mercury-liquid interface provides an ideal model for the simulation of charged conditions on a colloidal surface and the electrokinetic phenomenon of colloidal particles. A mercury drop is electrically conductive and can have a flow field inside. Due to these specific properties many phenomena of rigid colloidal particles are pronounced in the case of mercury drops. It was observed that when an electric

\footnotetext{
* Corresponding author.

E-mail address: jphsu@ccms.ntu.edu.tw (J.-P. Hsu).
}

current is applied on a mercury drop placed in a capillary, the potential drop at the mercury-liquid interface varies with the current [1]. If the mercury drop is positively charged, its surface tension near the cathode is larger than that near the anode, and therefore, it moves toward the cathode, the so-called electrocapillary motion. Other experimental work that involves a mercury-liquid interface, can also be found in standard textbooks [2-4]. The charged condition on a mercury surface, the adsorption of molecules on the surface, and the structure of the double layer are investigated. The theoretical analysis of the motion of a mercury drop in an electric field was originated by Craxford et al. [1]. It was concluded that the force experienced by a mercury drop could be expressed as the product of the strength of the electric field and the amount of charges in the electrical double layer surrounding the drop. Although this is incorrect when the total amount of charges vanishes, the result derived under the condition of low potential gradient provides valuable reference for electrophoresis. Levich and Frumkin investigated the electrokinetic phenomenon of a charged mercury drop under the condition of a thin double layer by solving the Lippmann equation subject to some simplified boundary conditions on a mercury surface [1]. Ohshima et al. [5] analyzed theoretically the electrokinetic 
phenomenon of a dilute mercury drop. Their analysis was based on the following assumptions: (i) The flow fields inside and outside of a mercury drop are in the creeping flow regime. (ii) The electric field and the gravity are weak so that both the mobility and the sedimentation velocity are directly proportional to them. (iii) The surface tension is high so that a mercury drop remains spherical. (iv) The surface of a mercury drop is impenetrable to ions. The numerical scheme of O'Brien and White [6] was used to solve the governing equations. They were able to show that an Onsager relation [7] can be recovered under the conditions of low surface potential and negligible double-layer overlapping. The analysis was extended by Ohshima [8] to the case of a concentrated dispersion by adopting the unit cell model of Kuwabara [9]. Assuming low surface potential and negligible double-layer overlapping Ohshima was able to derive an analytical expression for the electrophoretic mobility, and showed that it reduced to the mobility of a rigid sphere as the viscosity of a drop approaches infinity. It was found that if $\kappa a$ is large, the mobility approaches a constant, which is independent of the volume fraction of the spheres.

In the present study, the electrophoretic behavior of a concentrated dispersion of mercury drops is investigated under the conditions of arbitrary surface potential and double-layer thickness. In addition, the effects of doublelayer polarization and the overlapping of adjacent doublelayers on the system under consideration are examined.

\section{Theory}

We consider a uniform dispersion of mercury drops of radius $a$ in a $z_{1}: z_{2}$ electrolyte solution, $z_{1}$ and $z_{2}$ being respectively the valences of cations and anions. Referring to Fig. 1, the unit cell model of Kuwabara [9] is adopted to simulate the behavior of the system under consideration. Here, each mercury drop is enclosed by a concentric spherical liquid shell of radius $b$, the physical properties of which are the same as those of the dispersion medium. A uniform electric field is applied in the $Z$ direction, and the mercury drop moves in the same direction. The spherical coordinates $(r, \theta, \varphi)$ are chosen with its origin located at the center of the representative particle. The governing equations include those for flow field, electrical field, and concentration.

Suppose that the liquid phase is incompressible, and the flow field can be described by the Navier-Stokes equation. For flow fields outside the mercury drop we have

$\eta \nabla^{2} \boldsymbol{v}-\nabla p-\rho \nabla \phi=0$,

$\nabla \cdot \boldsymbol{v}=0$

where $p$ is pressure, $\boldsymbol{v}$ is velocity, and $\eta$ and $\rho$ are respectively viscosity and space charge density. For the flow field inside the mercury drop, we have

$\eta_{d} \nabla^{2} \boldsymbol{v}_{I}-\nabla p_{I}=0$

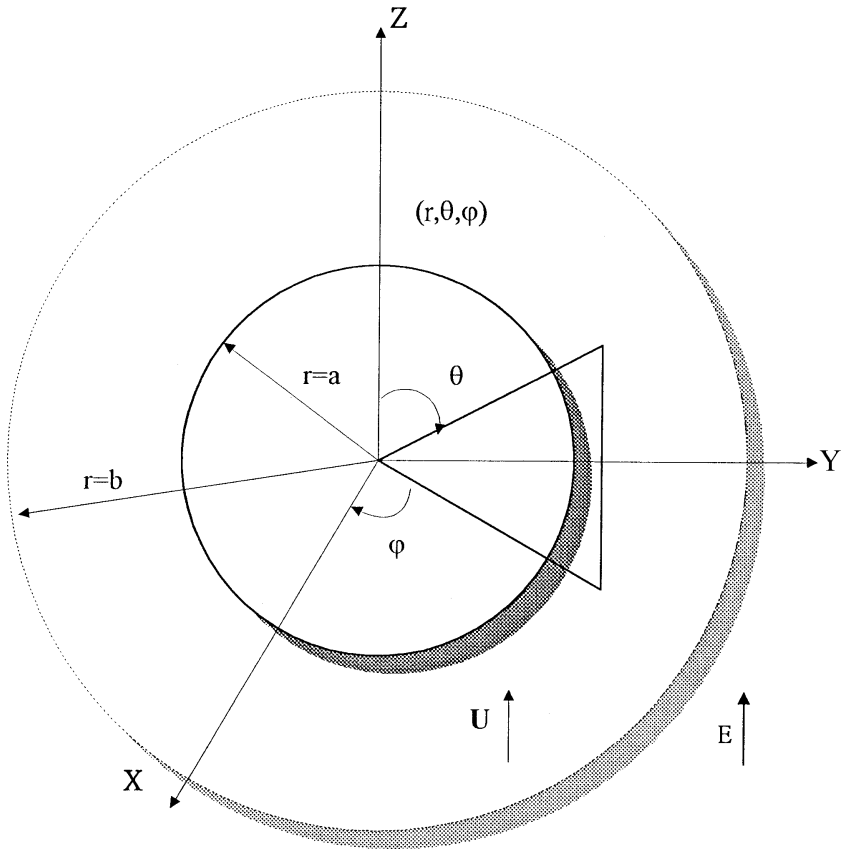

Fig. 1. Schematic representation of the system under consideration. A representative mercury drop of radius $a$ is enclosed by a liquid spherical shell of radius $b$. An electric field $\boldsymbol{E}$ is applied in the $Z$ direction.

$\nabla \cdot v_{I}=0$

where $\eta_{d}$ is the viscosity of mercury.

We assume that the electrical field can be described by the Poisson equation

$\nabla^{2} \phi=-\frac{\rho}{\varepsilon}$,

$\rho=\sum_{j} n_{j} z_{j} e$,

where $\nabla$ is the differential operator in spherical coordinates, and $\varepsilon$ and $\rho$ are respectively the permittivity of the dispersion medium and the space charge density. $n_{j}$ is the number concentration of ionic species $j$ which is further assumed to follow the Boltzmann distribution, that is,

$n_{j}=n_{j 0} \exp \left(-\frac{z_{j} e \phi}{k_{B} T}\right)$,

where $n_{j 0}$ is the bulk number concentration of ionic species $j, \phi$ is the electrical potential, $k_{B}$ is the Boltzmann constant, and $T$ is the absolute temperature.

The conservation of the amount of ionic species yields

$\nabla \cdot \boldsymbol{f}_{j}=0$,

where the flux of the $j$ th ionic species $\boldsymbol{f}_{j}$ can be expressed as

$\boldsymbol{f}_{j}=-D_{j}\left[\nabla n_{j}+\frac{z_{j} e n_{j}}{k_{B} T} \nabla \phi\right]+n_{j} \boldsymbol{v}$,

where $D_{j}, n_{j}$, and $z_{j}$ are respectively the diffusion coefficient, number concentration, and the valence of ionic species 
$j$, and $e$ is the elementary charge. Substituting this expression into Eq. (8) gives

$$
\begin{gathered}
\nabla^{2} n_{j}+\frac{z_{j} e}{k_{B} T}\left(\nabla n_{j} \cdot \nabla \phi+n_{j} \nabla^{2} \phi\right) \\
-\frac{1}{D_{j}} \mathbf{v} \cdot \nabla n_{j}=0 .
\end{gathered}
$$

The electrical potential comprises two basic elements: the potential in the absence of an applied electric field, that is, the equilibrium potential, $\phi_{1}$, and a perturbed potential due to the presence of the applied electric field, $\phi_{2}$. The distribution of ions is influenced by the flow field, the effect of double-layer polarization. This can be simulated by considering a perturbed term $g_{j}$ in the electrical potential [6]. Therefore, Eq. (7) becomes

$n_{j}=n_{j 0} \exp \left[-\frac{z_{j} e\left(\phi_{1}+\phi_{2}+g_{j}\right)}{k_{B} T}\right]$,

and $\phi_{1}$ and $\phi_{2}$ satisfy, respectively,

$\nabla^{2} \phi_{1}=-\sum_{j=1}^{N} \frac{z_{j} e n_{j 0}}{\varepsilon} \exp \left(-\frac{z_{j} e \phi_{1}}{k_{B} T}\right)$

and

$$
\begin{aligned}
\nabla^{2} \phi_{2}= & \nabla^{2} \phi-\nabla^{2} \phi_{1} \\
= & \sum_{j=1}^{2} \frac{z_{j} e n_{j 0}}{\varepsilon} \exp \left(-\frac{z_{j} e\left(\phi_{1}+\phi_{2}+g_{j}\right)}{k_{B} T}\right) \\
& -\sum_{j=1}^{2} \frac{z_{j} e n_{j 0}}{\varepsilon} \exp \left(-\frac{z_{j} e \phi_{1}}{k_{B} T}\right) .
\end{aligned}
$$

The governing equations for the flow field can be simplified by taking the curl on both Eqs. (1) and (3), applying the condition expressed in Eq. (2), and introducing the stream function $\Psi$. We have the following relations:

$v_{r}=-\frac{1}{r^{2} \sin \theta} \frac{\partial \Psi}{\partial \theta}$,

$v_{\theta}=\frac{1}{r \sin \theta} \frac{\partial \Psi}{\partial r}$

where $v_{r}$ and $v_{\theta}$ are respectively the $r$ and $\theta$ components of the velocity. A similar approach can also be employed to the flow field inside a mercury drop. We arrive at

$E^{4} \Psi=-\frac{1}{\eta} \sin \theta \cdot \nabla \times\left[\rho \nabla\left(\phi_{1}+\phi_{2}\right)\right]$,

outside the mercury drop,

$E^{4} \Psi_{I}=0, \quad$ inside the mercury drop,

where $E^{4}=E^{2} E^{2}$ with

$E^{2}=\frac{\partial^{2}}{\partial r^{2}}+\frac{\sin \theta}{r^{2}} \frac{\partial}{\partial \theta}\left(\frac{1}{\sin \theta} \frac{\partial}{\partial \theta}\right)$.
The governing equation for the concentration field becomes, taking the effect of double-layer relaxation into account,

$$
\begin{aligned}
\nabla^{2} g_{j}-\frac{z_{j} e}{k_{B} T} \nabla \phi_{1} \cdot \nabla g_{j} \\
=\frac{1}{D_{j}} u \cdot \nabla \phi+\frac{1}{D_{j}} u \cdot \nabla g_{j}+\frac{z_{j} e}{k_{B} T} \nabla \phi_{2} \cdot \nabla g_{j} \\
\quad+\frac{z_{j} e}{k_{B} T} \nabla g_{j} \cdot \nabla g_{j} .
\end{aligned}
$$

\subsection{Boundary conditions}

\subsubsection{Flow field}

Suppose that both the velocity and the shear stress are continuous at the mercury-liquid interface. The boundary conditions for the flow field are assumed to be

$$
\begin{aligned}
& \left.\boldsymbol{v}\right|_{r=a^{+}}=\left.\boldsymbol{v}_{I}\right|_{r=a^{-}}, \\
& (\boldsymbol{\tau} \cdot \boldsymbol{n}) \times\left.\boldsymbol{n}\right|_{r=a^{+}}=\left(\boldsymbol{\tau}_{I} \cdot \boldsymbol{n}\right) \times\left.\boldsymbol{n}\right|_{r=a^{-}}, \\
& \left.\boldsymbol{v} \cdot \boldsymbol{n}\right|_{r=a^{+}}=\left.\boldsymbol{v}_{I} \cdot \boldsymbol{n}\right|_{r=a^{-}}=0,
\end{aligned}
$$

where the subscript $I$ denotes the mercury phase and $\boldsymbol{n}$ is the unit normal vector. The second condition can also be expressed as $\left.\tau_{r \theta}\right|_{r=a^{+}}=\left.\tau_{r \theta, I}\right|_{r=a^{-}}$. The last condition implies that there is no mass change between the mercury phase and the liquid phase.

The following two conditions are assumed at the virtue surface, the outer boundary of a representative Kuwabara cell:

$\left.\boldsymbol{v} \cdot \boldsymbol{n}\right|_{r=b^{-}}=-U \cos \theta \quad$ and $\quad \boldsymbol{\nabla} \times \boldsymbol{v}=\mathbf{0}, \quad r=b$.

\subsubsection{Electrical field}

We assume the following [8]: (i) a mercury drop remains spherical, (ii) the mercury-liquid interface is impenetrable to ions, (iii) the interior of a mercury drop is free of ions, (iv) the mercury-liquid interface remains constant electrical potential. Assumption (i) is based on the high surface tension of mercury. The rest of the assumptions were proposed by Levich [1]. The boundary conditions for $\phi_{1}$ are assumed to be

$\phi_{1}=\varsigma_{a}, \quad r=a$,
$\frac{\partial \phi_{1}}{\partial r}=0, \quad r=b$,

where $\zeta_{a}$ is the zeta potential of a mercury drop. The first condition suggests that the mercury drop remains at a constant surface potential. The second condition implies that a unit cell as a whole is in electroneutrality, and there is no current between adjacent unit cells.

The boundary conditions for $\phi_{2}$ are assumed to be

$$
\begin{aligned}
& \frac{\partial \phi_{2}}{\partial r}=0, \quad r=a, \\
& \frac{\partial \phi_{2}}{\partial r}=-E_{z} \cos \theta, \quad r=b .
\end{aligned}
$$


The first one states that the mercury surface is impenetrable to ionic species in dispersion medium, and the second one defines the magnitude of the applied electric field on the virtual surface.

\subsubsection{Concentration field}

The surface of a mercury drop is impenetrable to ions and the concentration of ions reaches the equilibrium value at the virtue surface. Therefore, the boundary conditions for the concentration field are

$\boldsymbol{f}_{j} \cdot \boldsymbol{n}=\boldsymbol{f}_{j} \cdot \boldsymbol{r}=0, \quad r=a$,

$n_{j}=n_{j 0}, \quad r=b$.

\subsection{Scaled governing equations}

For a simpler mathematical treatment, the scaled quantities are used in the following analysis. The radius of a mercury drop $a$ is chosen as the length scale, the equilibrium surface potential $\zeta_{a}$ is chosen as the scale factor for potential, and the bulk concentration of electrolyte $n_{j 0}$ is chosen as the scale factor for concentration. Let $n_{10}$ and $n_{20}$ be the bulk concentrations of cations and anions. Then the electroneutrality requires that $n_{20}=n_{10} / \alpha$ with $z_{2} / z_{1}=-\alpha$. We have the relation

$n_{10} z_{1}=\frac{(\kappa a)^{2} \varepsilon k_{B} T}{(1+\alpha) e^{2} a^{2} z_{1}}$.

We define $\nabla^{*}=a \nabla, \phi_{1}=\phi_{1}^{*} \varsigma a, \phi_{2}=\phi_{2}^{*} \varsigma_{a}, n_{j}=n_{j}^{*} n_{j 0}$, $\phi_{r}=\varsigma_{a} z_{1} e / k_{B} T$, and $\lambda=a / b$, where the Debye length $(1 / \kappa)$ is defined by $1 / \kappa=\left[\varepsilon k_{B} T / \sum n_{j 0}\left(e z_{j}\right)^{2}\right]$. The parameter $\lambda$ is a measure for the concentration of mercury drops in the dispersion, and $\phi_{r}$ can be viewed as the relative magnitudes between the surface potential and $k_{B} T / z_{1} e$. Equation (12) can be rewritten as

$\nabla^{* 2} \phi_{1}^{*}=-\frac{1}{(1+\alpha)} \frac{(\kappa a)^{2}}{\phi_{r}}\left(n_{1}^{*}-n_{2}^{*}\right)$

where $n_{1}^{*}=\exp \left[-\phi_{r}\left(\phi_{1}^{*}+\phi_{2}^{*}+g_{1}^{*}\right)\right]$ and $n_{2}^{*}=\exp \left[-\phi_{r} \times\right.$ $\left.\left(\phi_{1}^{*}+\phi_{2}^{*}+g_{2}^{*}\right)\right]$.

The corresponding boundary conditions for $\phi_{1}^{*}$ are

$\phi_{1}^{*}=1, \quad r^{*}=1$

$\frac{\partial \phi_{1}^{*}}{\partial r^{*}}=0, \quad r^{*}=1 / \lambda$.

Similarly, Eq. (13) can be rewritten as

$$
\begin{aligned}
\nabla^{* 2} \phi_{2}^{*}= & -\frac{1}{(1+\alpha)} \frac{(\kappa a)^{2}}{\phi_{r}}\left\{\left(n_{1}^{*}-n_{2}^{*}\right)\right. \\
& \left.-\left[\exp \left(-\phi_{r} \phi_{1}^{*}\right)-\exp \left(\alpha \phi_{r} \phi_{1}^{*}\right)\right]\right\}
\end{aligned}
$$

and the corresponding boundary conditions become

$\partial \phi_{2}^{*} / \partial r^{*}=0, \quad r^{*}=1$,

$\partial \phi_{2}^{*} / \partial r^{*}=-E_{z}^{*} \cos \theta, \quad r^{*}=1 / \lambda$,

where $E_{z}^{*}=E_{z} a / \varsigma_{a}$.
Let $v^{*}=v / U_{E}$ where $U_{E}=\varepsilon \varsigma_{a}^{2} / \eta a$ is the electrophoretic velocity derived by Smoluchowski [10] for the case an electric field of strength $\left(\zeta_{a} / a\right)$ is applied. The scaled form of Eq. (19) can be written as

$$
\begin{aligned}
& \nabla^{* 2} g_{1}^{*}-\phi_{r} \nabla^{*} \phi_{1}^{*} \cdot \nabla g_{1}^{*}=P e_{1} u^{*} \cdot \nabla \phi_{1}^{*}+P e_{1} u^{*} \cdot \nabla \phi_{2}^{*} \\
& +P e_{1} u^{*} \cdot \nabla g_{1}^{*}+\phi_{r} \nabla^{*} \phi_{2}^{*} \cdot \nabla^{*} g_{1}^{*}+\phi_{r} \nabla^{*} g_{1}^{*} \cdot \nabla^{*} g_{1}^{*} .
\end{aligned}
$$

The corresponding boundary conditions become

$$
\begin{aligned}
& \partial g_{1}^{*} / \partial r^{*}=0, \quad r^{*}=1, \\
& g_{1}^{*}=-\phi_{2}^{*}, \quad r^{*}=1 / \lambda .
\end{aligned}
$$

Similarly,

$$
\begin{aligned}
& \nabla^{* 2} g_{2}^{*}+\alpha \phi_{r} \nabla^{*} \phi_{1}^{*} \cdot \nabla g_{2}^{*} \\
& =P e_{2} u^{*} \cdot \nabla^{*} \phi_{1}^{*}+P e_{2} u^{*} \cdot \nabla \phi_{2}^{*}+P e_{2} u^{*} \cdot \nabla g_{2}^{*} \\
& \quad-\alpha \phi_{r} \nabla^{*} \phi_{2}^{*} \cdot \nabla^{*} g_{2}^{*}-\alpha \phi_{r} \cdot \nabla^{*} g_{2}^{*} \nabla^{*} g_{2}^{*}, \\
& \partial g_{2}^{*} / \partial r^{*}=0, \quad r^{*}=1, \\
& g_{2}^{*}=-\phi_{2}^{*}, \quad r^{*}=1 / \lambda .
\end{aligned}
$$

In these expressions $P e_{j}=U_{E} a / D_{j}$ is the Peclet number of species $j$.

The scaled stream function $\Psi^{*}$ is defined as $\Psi^{*}=$ $\psi / U_{E} a$, and the scaled space charge density $\rho^{*}$ as $\rho^{*}=$ $\rho / \rho_{0}$, where $\rho_{0}=\varepsilon \kappa^{2} \varsigma_{a} /(1+\alpha) \phi_{r}$ and $\rho^{*}=n_{1}^{*}-n_{2}^{*}$. Therefore Eq. (16) can be rewritten as

$$
\begin{aligned}
E^{* 4} \Psi^{*}= & -\frac{(\kappa a)^{2}}{(1+\alpha)}\left[\left(\frac{\partial g_{1}^{*}}{\partial r^{*}} n_{1}^{*}+\frac{\partial g_{2}^{*}}{\partial r^{*}}\left(\alpha n_{2}^{*}\right)\right) \frac{\partial \phi^{*}}{\partial \theta}\right. \\
& \left.-\left(\frac{\partial g_{1}^{*}}{\partial r^{*}} n_{1}^{*}+\frac{\partial g_{2}^{*}}{\partial r^{*}}\left(\alpha n_{2}^{*}\right)\right) \frac{\partial \phi^{*}}{\partial r^{*}}\right] \sin \theta .
\end{aligned}
$$

Equation (17) can be rewritten as

$E^{* 4} \Psi_{I}^{*}=0$.

It can be shown that

$\Psi^{*}=0$,

$a \frac{\partial^{2} \Psi^{*}}{\partial^{*} r^{* 2}}-2 \frac{\partial \Psi^{*}}{\partial^{*} r^{*}}+\frac{1}{a} \Psi^{*}-\frac{3 \eta_{d}}{\eta} \frac{\partial \Psi^{*}}{\partial^{*} r^{*}}=0, \quad r^{*}=1$,

$\Psi^{*}=\frac{1}{2} U^{*} r^{* 2} \sin ^{2} \theta$,

$\left[\frac{1}{r^{*}} \frac{\partial^{2}}{\partial^{*} r^{* 2}}-\frac{2}{r^{* 3}}\right] \Psi^{*}=0, \quad r^{*}=1 / \lambda$.

Following the treatment of O'Brien and White [6], we consider the case when the applied electric field is relatively weak compared with that induced by mercury drops. In this case, we assume that $\phi_{2}^{*}, g_{1}^{*}$, and $g_{2}^{*}$ are much smaller than $\phi_{1}^{*}$, and $n_{1}^{*}=\exp \left[-\phi_{r}\left(\phi_{1}^{*}+\phi_{2}^{*}+g_{1}^{*}\right)\right]$ and $n_{2}^{*}=\exp \left[\alpha \phi_{r}\left(\phi_{1}^{*}+\phi_{2}^{*}+g_{2}^{*}\right)\right]$ can be approximated respectively by

$n_{1}^{*}=\exp \left(-\phi_{r} \phi_{1}^{*}\right)\left[1-\phi_{r}\left(\phi_{2}^{*}+g_{1}^{*}\right)\right]$ 
and

$n_{2}^{*}=\exp \left(\alpha \phi_{r} \phi_{1}^{*}\right)\left[1+\alpha \phi_{r}\left(\phi_{2}^{*}+g_{2}^{*}\right)\right]$.

Equations (32), (37), (40), and (43) can be linearized based on these expressions and neglecting $-\phi_{r}\left(\phi_{2}^{*}+g_{1}^{*}\right)$ and $\alpha \phi_{r}\left(\phi_{2}^{*}+g_{2}^{*}\right)$, and the products terms involving perturbation terms.

Equation (31) can be rewritten as

$\nabla^{* 2} \phi_{1}^{*}=-\frac{1}{(1+\alpha)} \frac{(\kappa a)^{2}}{\phi_{r}}\left(\exp \left(-\phi_{r} \phi_{1}^{*}\right)-\exp \left(\alpha \phi_{r} \phi_{1}^{*}\right)\right)$.

Equation (34) can be approximated by the linear expression

$$
\begin{gathered}
\nabla^{* 2} \phi_{2}^{*}-\frac{(k a)^{2}}{1+\alpha}\left(\exp \left(-\phi_{r} \phi_{1}^{*}\right)+\alpha \exp \left(\alpha \phi_{r} \phi_{1}^{*}\right)\right) \phi_{2}^{*} \\
=\frac{(k a)^{2}}{1+\alpha}\left(\exp \left(-\phi_{r} \phi_{1}^{*}\right) g_{1}^{*}+\alpha \exp \left(\alpha \phi_{r} \phi_{1}^{*}\right) g_{2}^{*}\right) .
\end{gathered}
$$

Equations (37) and (40) can be rewritten respectively as

$\nabla^{* 2} g_{1}^{*}-\phi_{r} \nabla^{*} \phi_{1}^{*} \cdot \nabla^{*} g_{1}^{*}=P e_{1} u^{*} \cdot \nabla^{*} \phi_{1}^{*}$

and

$\nabla^{* 2} g_{2}^{*}+\alpha \phi_{r} \nabla^{*} \phi_{1}^{*} \cdot \nabla^{*} g_{2}^{*}=P e_{2} u^{*} \cdot \nabla^{*} \phi_{1}^{*}$.

Equation (43) can be expressed as

$E^{* 4} \Psi^{*}=\frac{(\kappa a)^{2}}{(1+\alpha)}\left[\left(\frac{\partial g_{1}^{*}}{\partial \theta^{*}} n_{1}^{*}+\frac{\partial g_{2}^{*}}{\partial \theta^{*}}\left(\alpha n_{2}^{*}\right)\right) \frac{\partial \phi^{*}}{\partial r^{*}}\right] \sin \theta$

The symmetric nature of the problem under consideration suggests that the dependent variables are $\varphi$-independent. Also, the governing equations are all linear, and can be solved by the method of separation of variables. It can be shown that the solution of Eqs. (49) to (53) can be expressed respectively as $\phi_{2}^{*}=\Phi_{2}(r) \cos \theta, g_{1}^{*}=G_{1}(r) \cos \theta, g_{2}^{*}=$ $G_{2}(r) \cos \theta$, and $\Psi^{*}=\bar{\Psi}(r) \sin ^{2} \theta$. It can be shown that the governing equation for $\Phi_{2}$ takes the form

$$
\begin{array}{r}
L \Phi_{2}-\frac{(\kappa a)^{2}}{1+\alpha}\left[\exp \left(-\phi_{r} \phi_{1}^{*}\right)+\alpha \exp \left(\alpha \phi_{r} \phi_{1}^{*}\right)\right] \Phi_{2} \\
=\frac{(\kappa a)^{2}}{1+\alpha}\left[\exp \left(-\phi_{r} \phi_{1}^{*}\right) G_{1}+\alpha \exp \left(\alpha \phi_{r} \phi_{1}^{*}\right) G_{2}\right]
\end{array}
$$

where

$$
L \equiv \frac{d^{2}}{d r^{* 2}}+\frac{2}{r^{*}} \frac{d}{d r^{*}}-\frac{1}{r^{* 2}} .
$$

The associated boundary conditions are

$$
\begin{aligned}
& \Phi_{2}=0, \quad r^{*}=1, \\
& \Phi_{2}=-E_{z}^{*}, \quad r^{*}=1 / \lambda .
\end{aligned}
$$

The governing equation for $G_{1}$ is

$$
L G_{1}-\phi_{r}^{2} \frac{d \phi_{1}^{*}}{d r^{*}}=P e_{1} \cdot \phi_{r}^{2} v_{r}^{*} \frac{d \phi_{1}^{*}}{d r^{*}}
$$

The associated boundary conditions are

$$
\begin{aligned}
& G_{1}=0, \quad r^{*}=1, \\
& G_{1}=-\Phi_{2}, \quad r^{*}=1 / \lambda .
\end{aligned}
$$

The governing equation for $G_{2}$ is

$L G_{2}+\alpha \phi_{r}^{2} \frac{d \phi_{1}^{*}}{d r^{*}}=P e_{2} \cdot \phi_{r}^{2} v_{r}^{*} \frac{d \phi_{1}^{*}}{d r^{*}}$.

The associated boundary conditions are

$G_{2}=0, \quad r^{*}=1$,

$G_{2}=-\Phi_{2}, \quad r^{*}=1 / \lambda$.

The governing equation for $\bar{\Psi}$ can be expressed as

$D^{4} \bar{\Psi}=-\frac{(\kappa a)^{2}}{1+\alpha}\left[\left(n_{1}^{*} G_{1}+n_{2}^{*} G_{2}\right) \frac{d \phi_{1}^{*}}{d r^{*}}\right]$,

where $D^{4} \equiv D^{2} D^{2}=\left(d^{2} / d r^{* 2}-2 / r^{* 2}\right)^{2}$. The associated boundary conditions are

$\bar{\Psi}=0$,

$a \frac{d^{2} \bar{\Psi}}{d r^{* 2}}-2 \frac{d \bar{\Psi}}{d^{*} r^{*}}+\frac{1}{a} \bar{\Psi}-\frac{3 \eta_{d}}{\eta} \frac{d \bar{\Psi}}{d r^{*}}=0, \quad r^{*}=1$,

$\bar{\Psi}=0$,

$\left[\frac{1}{r^{*}} \frac{d^{2}}{d^{*} r^{* 2}}-\frac{2}{r^{* 3}}\right] \bar{\Psi}=0, \quad r^{*}=1 / \lambda$.

\subsection{Electrophoretic mobility}

Following the approach of O'Brien and White [6], the problem under consideration is decomposed into two subproblems. The first problem considers the motion of a mercury drop in the absence of the applied electric field, and the second problem considers the phenomenon of a stationary mercury drop in the applied electric field. The flow field and the electric field can be obtained by summing the corresponding results of these two problems. In the first problem the drag force exerted on the surface of a mercury drop by the surrounding fluid, $f_{1}$, is proportional to the terminal velocity of the mercury drop, that is, $f_{1}=\delta U^{*}$, where the scaled terminal velocity $U^{*}$ is defined as $U^{*}=U / U_{E}$. In the second problem the force exerted on the surface of a mercury drop due to presence of the applied electric field, $f_{2}$, is proportional to the strength of the applied electric field, that is, $f_{2}=\beta E_{z}^{*}$, where $E_{z}^{*}$ is the scaled strength of the applied electric field. We define the scaled mobility $U_{m}^{*}$ as $U_{m}^{*}=U^{*} / E^{*}$. At steady state, the sum of the forces in problems 1 and 2 vanishes, and we have $U_{m}^{*}=U^{*} / E^{*}=-\beta / \delta$.

Note that $f_{1}$ corresponds to the scaled drag force $F_{D z}$. According to Happel and Brenner [12], this force can be expressed as

$$
F_{D z}=\eta \pi \int_{0}^{\pi}\left(r^{4} \sin ^{3} \theta \frac{\partial}{\partial r} \frac{E^{2} \psi}{r^{2} \sin ^{2} \theta}\right)_{r=a} d \theta
$$




$$
-\pi \int_{0}^{\pi}\left(r^{2} \sin ^{2} \theta \rho \frac{\partial \phi}{\partial \theta}\right)_{r=a} d \theta .
$$

For the present case, it can be shown that

$$
\begin{aligned}
F_{D z}= & \pi \varepsilon \varsigma_{a}^{2} \int_{0}^{\pi}\left[r^{* 4} \sin ^{3} \theta \frac{\partial}{\partial r^{*}} \frac{E^{* 2} \Psi^{*}}{r^{* 2} \sin ^{2} \theta}\right]_{r^{*}=1} d \theta \\
& -\pi \varepsilon \varsigma_{a}^{2} \frac{(\kappa a)^{2}}{(1+\alpha) \phi_{r}} \\
& \times \int_{0}^{\pi}\left[r^{* 2} \sin ^{2} \theta\left(n_{1}^{*}-n_{2}^{*}\right) \frac{\partial \phi_{2}^{*}}{\partial \theta}\right]_{r^{*}=1} d \theta \\
= & \pi \varepsilon \zeta_{a}^{2}\left(U^{*} K_{D f}-\frac{(\kappa a)^{2}}{(1+\alpha) \phi_{r}} E^{*} K_{D e}\right),
\end{aligned}
$$

where $U^{*} K_{D f}$ and $E^{*} K_{D e}$ denote respectively the first and second integrals on the right-hand side of the first expression of Eq. (68).

The scaled force $F_{E z}$ can be evaluated by

$$
F_{E z}=\iint_{S} \sigma(-\nabla \phi) d A,
$$

where $\sigma$ denotes the surface charge density. In spherical coordinates, we have

$$
\begin{aligned}
F_{E z}= & 2 \pi \varepsilon \zeta_{a}^{2} \int_{0}^{\pi}\left(\frac{\partial \phi_{1}^{*}}{\partial r^{*}}\right)_{r^{*}=1} \\
& \times\left(\frac{\partial\left(\phi_{1}^{*}+\phi_{2}^{*}\right)}{\partial r^{*}} \cos \theta-\frac{1}{r^{*}} \frac{\partial\left(\phi_{1}^{*}+\phi_{2}^{*}\right)}{\partial r^{*}} \sin \theta\right)_{r^{*}=1} \\
& \times r^{* 2} \sin \theta d \theta \\
= & 2 \pi \zeta_{a}^{2} E^{*} K_{E},
\end{aligned}
$$

where $E^{*} K_{E}$ is the integral of the right-hand side of the first expression of Eq. (70).

\section{Results and discussion}

The behaviors of the system under consideration are examined through numerical simulation. The governing equations are solved by applying the pseudo-spectral method based on Chebyshev polynomials [11]. This method is readily applicable for the present boundary-value problem, and has the merits of fast rate of convergence and the convergent properties independent of the associated boundary conditions. Figure 2 shows the variation of the scaled mobility $U_{m}^{*}$ as a function of $\kappa a$ at various scaled surface potentials of a mercury drop $\phi_{r}$ for the case $\lambda=0.5$, and that for a smaller $\lambda$ is illustrated in Fig. 3. The corresponding results of Ohshima [8] are also presented for comparison. Note that the definition of $\lambda(=a / b)$ suggests that it is a measure for the concentration of a mercury drop. The larger its value, the higher the concentration. Figure 2 reveals that for a low $\phi_{r}$, mobility increases monotonically with $\kappa a$. For medium and high $\phi_{r}$ the mobility curve has a reflection point for $\kappa a$ in the range 1 to 10 . It is interesting to note that if $\phi_{r}$ is high, the mobility curve exhibits a local minimum. A comparison between Figs. 2 and 3 reveals that this phenomenon is pronounced if $\lambda$ becomes larger, that is, a more concentrated dispersion. The reflection point of the mobility curve arises from the overlapping of adjacent double layers. For example, in the case of Fig. $2, \lambda=a / b=0.5$, that is, the thickness of the double layer surrounding a mercury drop is the same as the radius of the liquid shell surrounding it at $\kappa a=1$. This is consistent with the results presented in Fig. 2 in which the reflection point is in the range 1 to 2 . Figures 2 and 3 show that $U_{m}^{*}$ decreases with $\kappa a$, that is, the thicker the double

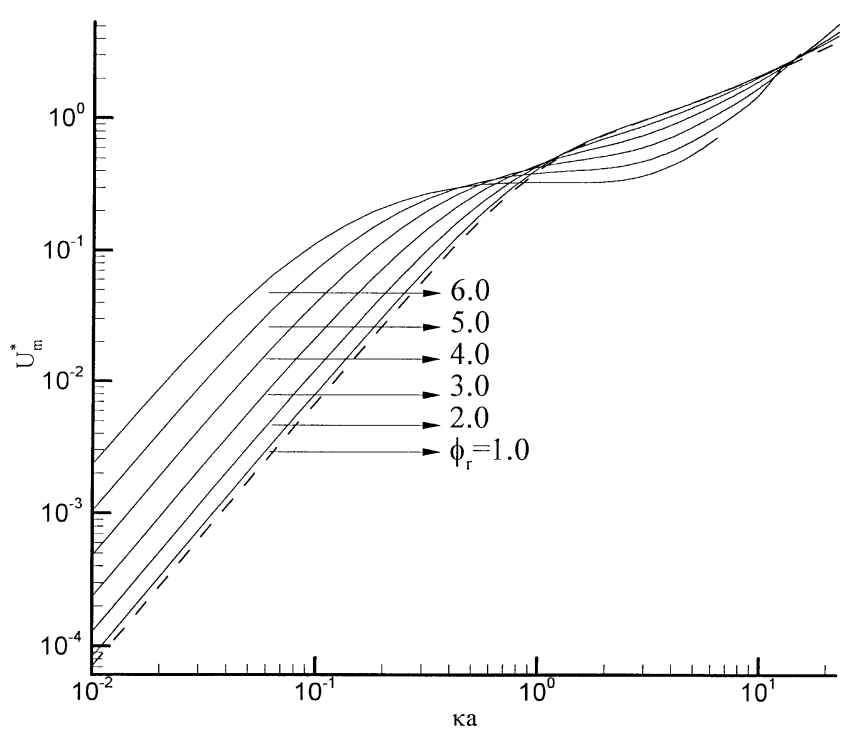

Fig. 2. Variation of scaled mobility $U_{m}^{*}$ as a function of $\kappa a$ at various scaled surface potentials of mercury drop $\phi_{r}$. The dashed line represents the result of Ohshima [8]. $\alpha=1, P e_{1}=P e_{2}=0.1, \lambda=0.5, \eta_{d}=1.526$ $\mathrm{mPa}, \eta=0.890 \mathrm{mPa}$.

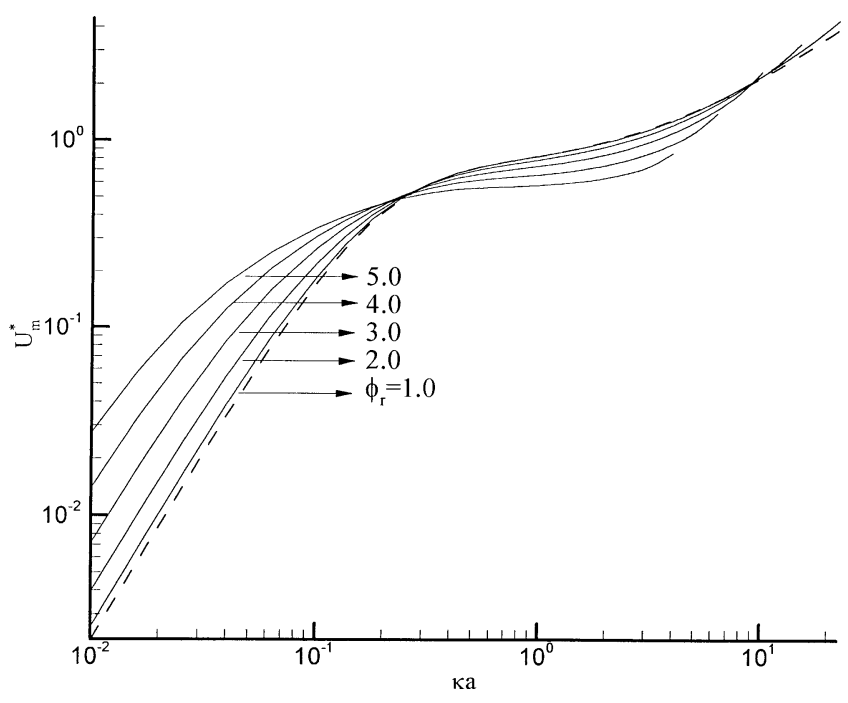

Fig. 3. Variation of scaled mobility $U_{m}^{*}$ as a function of $\kappa a$. Key: same as in Fig. 2 except that $\lambda=0.2$. 


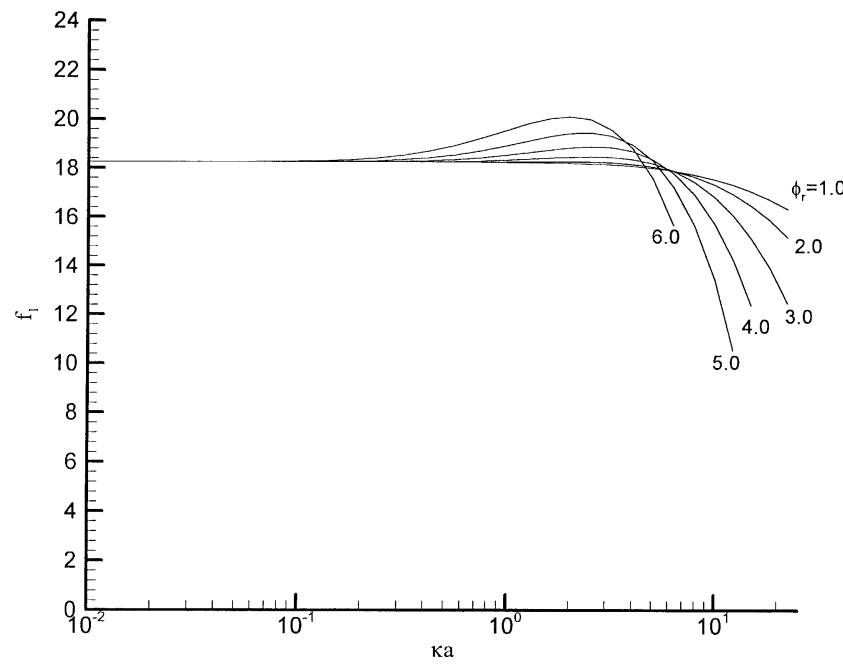

Fig. 4. Variation of scaled force in problem $1, f_{1}$ as a function of $\kappa a$ at various scaled surface potentials of mercury drop $\phi_{r}$ for the case of Fig. 2.

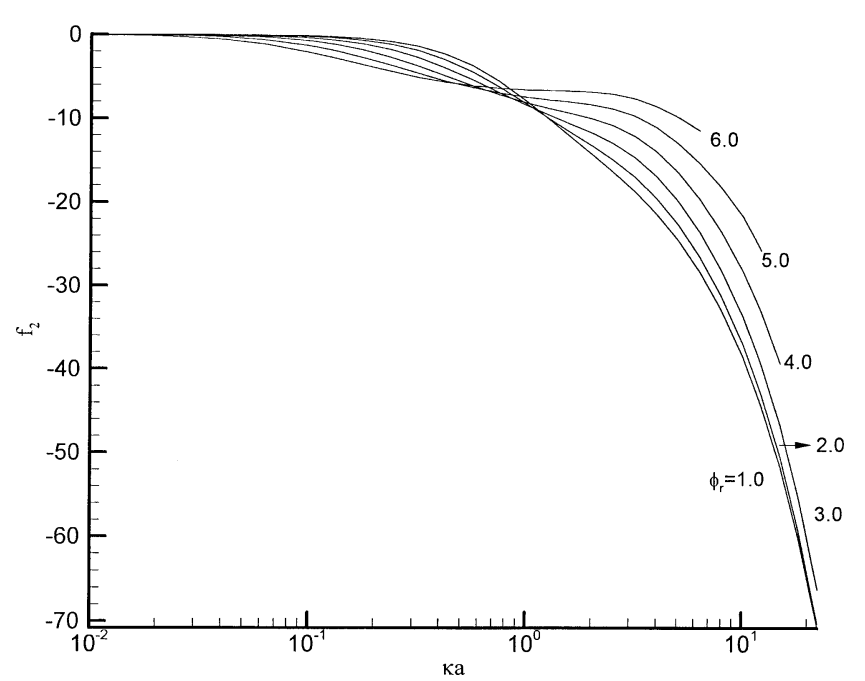

Fig. 5. Variation of scaled force in problem 2, $f_{2}$ as a function of $\kappa a$ at various scaled surface potentials of mercury drop $\phi_{r}$ for the case of Fig. 2.

layer the smaller the mobility. This is because if the double layer is thick, the electrical interaction between adjacent double layers becomes significant, which has the effect of lowering the mobility. Figures 2 and 3 also show that if $\kappa a$ is small (thick double layer), mobility increases with the surface potential of a mercury drop, and the reverse is true if $\kappa a$ is large (thin double layer). This can be explained by the relative magnitudes of the forces experienced by a mercury drop ins problems 1 and 2 . As mentioned previously, the scaled mobility can be expressed as $U_{m}^{*}=-f_{2} / f_{1}$. The variations of $f_{1}$ and $f_{2}$ as a function of $\kappa a$ for the case of Fig. 2 are illustrated in Figs. 4 and 5. A comparison between these figures shows that the variation of $f_{1}$ as $\phi_{r}$ varies is relatively less appreciable than that of $f_{2}$. According to Fig. 5, if $\kappa a$ is small, $\left|f_{2}\right|$ decreases with the decrease in $\phi_{r}$, and the reverse is true for a medium $\kappa a$.

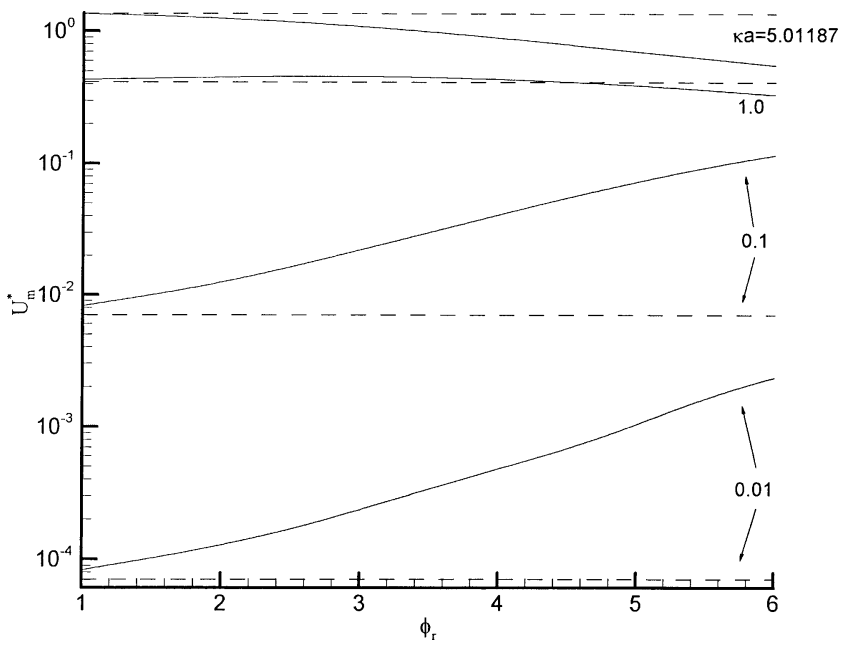

Fig. 6. Variation of scaled mobility $U_{m}^{*}$ as a function of scaled surface potentials of mercury drop $\phi_{r}$ at various $\kappa a$. The dashed line represents the result of Ohshima [8]. Key: same as Fig. 2.

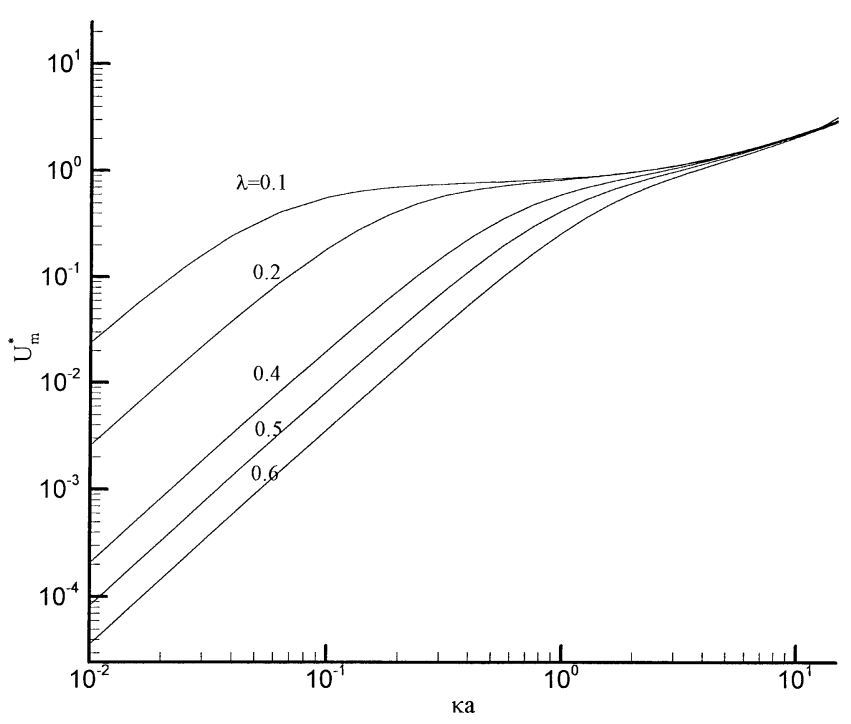

Fig. 7. Variation of scaled mobility $U_{m}^{*}$ as a function of $\kappa a$ at various $\lambda$. For the case $\alpha=1, P e_{1}=P e_{2}=0.1$, and $\phi_{r}=1.0$.

The variation in the scaled mobility $U_{m}^{*}$ as a function of the scaled surface potential of a mercury drop $\phi_{r}$ at various $\kappa a$ for the case of Fig. 2 is illustrated in Fig. 6. This figure reveals that the deviation of the linearized model of Ohshima becomes significant if the surface potential is high and the double layer is thick. In general, the result of Ohshima [8] is applicable for the case $\phi_{r}$ is lower than unity and $\kappa a$ is larger than 5.

Figure 7 shows the variation in scaled mobility $U_{m}^{*}$ as a function of $\kappa a$ at various concentrations of the dispersed phase measured by parameter $\lambda$. As can be seen from this figure, for a constant $\kappa a$, the larger the $\lambda$ the smaller the scaled mobility. This is expected since a large $\lambda$ implies a high concentration of the dispersed phase, which is disadvantageous to the movement of mercury drops. Figure 7 also reveals that as $\kappa a$ becomes large (i.e., thin double layer), 


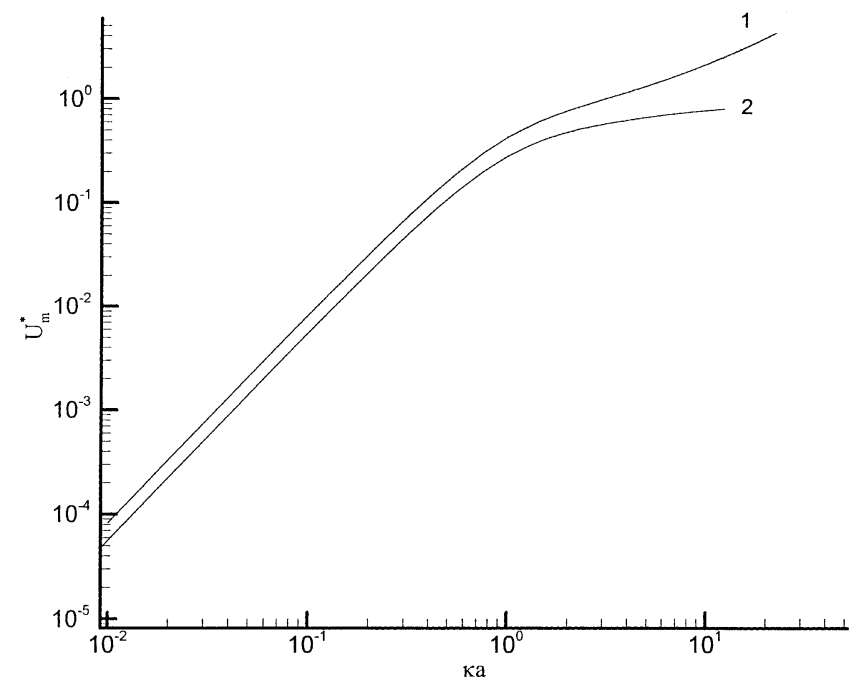

Fig. 8. Variation of scaled mobility $U_{m}^{*}$ as a function of $\kappa a$ at various $\lambda$. For the case $\alpha=1, P e_{1}=P e_{2}=0.1, \lambda=0.5$, and $\phi_{r}=1.0$. Curve 1 , mercury drop; curve 2, rigid particle.

the scaled mobility approaches a constant value, which is independent of the concentration of the dispersed phase. For rigid particles the scaled mobility should approach unity, and greater than unity for liquid drops.

A comparison between the scaled mobility $U_{m}^{*}$ evaluated by the present model and the corresponding result for the case of rigid particles is illustrated in Fig. 8. This figure reveals that the mobility of mercury drops is larger than that of the corresponding rigid particles. This is due mainly to the flow field inside a mercury drop. The flow field vanishes in the interior of a rigid entity, but this is not the case for a mercury drop. For the latter, a slip boundary condition needs to be employed at the surface of a mercury drop for the flow field. This implies that the tangential shear stress at the surface of a mercury drop is smaller than that of the corresponding rigid entity which leads to a larger mobility.

\section{Conclusion}

The objectives of the present study were to extend the analysis of previous study, which is limited to the case of low surface potential and neglecting the effects of double-layer polarization and the interaction between adjacent double layers. The results of numerical simulation can be summarized as follows: (i) For thin double layers the electrophoretic mobility increases with the thickness of the double layer, and the higher the surface potential the larger the mobility. But the reverse is true if the overlapping of adjacent double layers becomes significant. (ii) A higher concentration of the dispersed phase leads to a greater electrical repulsive force between adjacent mercury drops, and a smaller mobility. In this case, if the surface potential is sufficiently high, the mobility-versus- $\kappa a$ curve has a local minimum. (iii) For a fixed surface potential, the mobility approaches a constant value as $\kappa a$ becomes large which is independent of the concentration of the dispersed phase.

\section{Acknowledgment}

This work is supported by the National Science Council of the Republic of China.

\section{References}

[1] V.G. Levich, Physicochemical Hydrodynamics, Prentice-Hall, New York, 1962.

[2] P. Delahay, Double Layer and Electrode Kinetics, Interscience, New York, 1965.

[3] R.J. Hunter, Zeta Potential in Colloid Science, Academic Press, London, 1981.

[4] M.J. Sparnaay, in: D.H. Everett (Ed.), International Encyclopedia of Physics, Chemistry, and Chemical Physics, Vol. 4, Pergamon, Oxford, Topic 14.

[5] H. Ohshima, T.W. Healy, L.R. White, J. Chem. Soc. Faraday Trans. 280 (1984) 1643.

[6] R.W. O'Brien, J.R. White, J. Chem. Soc. Faraday Trans. 74 (1978) 1607.

[7] J.H. Masliyah, Electrokinetic Transport Phenomena, Alberta Oil Sands Technology and Research Authority, Edmonton, AB, 1994, p. 117.

[8] H. Ohshima, J. Colloid Interface Sci. 218 (1999) 535.

[9] S. Kuwabara, J. Phys. Soc. Jpn. 14 (1959) 527.

[10] M. Smoluchowski, Z. Phys. Chem. 93 (1918) 129.

[11] E. Lee, J.W. Chu, J.P. Hsu, J. Colloid Interface Sci. 205 (1998) 65.

[12] J. Happel, H. Brenner, Low Reynolds Number Hydrodynamics, Nijhoff, Dordrecht, 1983. 